\title{
Incorporation of Polymers into Calcined Clays as Improved Thermal Insulating Materials for Construction
}

\author{
Serina $\mathrm{Ng}^{1}$ and Bjørn Petter Jelle ${ }^{1,2}$ \\ ${ }^{1}$ Department of Materials and Structures, SINTEF Building and Infrastructure, 7465 Trondheim, Norway \\ ${ }^{2}$ Department of Civil and Transport Engineering, Norwegian University of Science and Technology (NTNU), \\ 7491 Trondheim, Norway \\ Correspondence should be addressed to Serina Ng; serina.ng@sintef.no
}

Received 30 March 2017; Revised 15 June 2017; Accepted 9 July 2017; Published 13 August 2017

Academic Editor: Andres Sotelo

Copyright (c) 2017 Serina Ng and Bjørn Petter Jelle. This is an open access article distributed under the Creative Commons Attribution License, which permits unrestricted use, distribution, and reproduction in any medium, provided the original work is properly cited.

Calcined clay is a Type Q supplementary cementing material according to EN197-1:2000. It possesses lower thermal conductivity than cement. To further improve its thermal insulation property, polymer-calcined clay complexes (PCCs) were produced in a onepot synthesis. Two contrasting polymers, polystyrene (PS) and polyethylene glycol (PEG), were employed. The hydrophilicity of the polymers influenced the thermal conductivity of PCC. Hydrophilic PEG entrapped more water molecules on clay layers than the hydrophobic PS, making PEG-PCC more thermally conducting than PS-PCC. Contaminants in calcined clays played a role in affecting the overall thermal conductivity. PCC can improve thermal insulation properties for future construction applications.

\section{Introduction}

Thermal insulation in buildings is an important factor for achieving thermal comfort for their occupants. This is particularly significant when large temperature gradients between indoor and outdoor climates are observed and high energy efficiency and low carbon footprint are of great importance [1,2]. Different thermal insulation composites have been produced, mainly based on reducing conduction and convection heat transfer through bulk insulators, or as radiant heat barriers, where positioning of building materials in relation to air spaces is optimized. In the last few years, our laboratory has focused on exploring the properties and the requirements of state-of-the-art thermal insulation materials and solutions for buildings, particularly dealing with the establishment of bulk thermal insulators from a bottom-up approach. Some materials that we have investigated include vacuum insulation panels (VIP) $[3,4]$, gas-filled panels (GFP) [5], aerogels [6], and also phase change materials (PCM) [7]. PCM by itself is not an insulation material in principle but may still be an important part of the thermal building envelope.
Composite materials are a common approach towards enabling thermal insulation and improved energy efficiency, one of which includes aerogel concrete systems where new thermal insulating concrete with structural properties is targeted $[8,9]$. There, instead of mainstream aerogel modifications, we have focused on matrix refinement, where one of the latest approaches targeted the inherent thermal properties of binders, specifically by partially replacing cement with a supplementary cementing material (SCM), calcined clay, to lower the overall thermal conductivity [10]. Calcined clay is produced by calcining clays at a temperature of between 600 and $1000^{\circ} \mathrm{C}$ [11]. Upon calcination, these clayey materials, both pure and contaminated ones, become pozzolanic in nature and can/may act as effective replacements of cement in the industry $[12,13]$. In the investigation, up to $20 \%$ improvement in the thermal conductivity was achieved when calcined clay was employed, while not compromising the mechanical properties of the concrete structures $[12,14]$. The results confirmed that designing new binder materials, for example, using calcined clay as SCM, was a step forward towards opening a new roadmap for designing insulating concrete in sustainable construction. Further improvement 


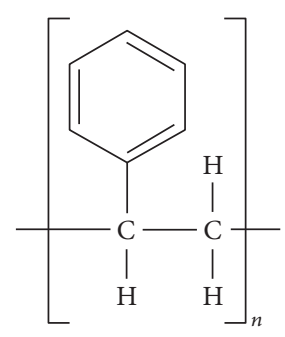

(a)

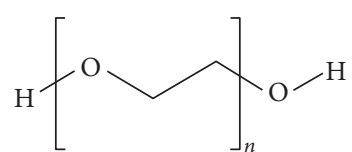

(b)
FIGURE 1: Chemical formulations of (a) polystyrene and (b) polyethylene glycol, respectively.

on the thermal conductivity of such binder materials, however, was needed to make them more desirable for actual applications as thermal insulators.

The purpose of this investigation was thus to further finetune the binder material, calcined clay. The starting material of calcined clay is clay, one of the most abundance materials all around the world that can incorporate and react with different ions and organics to possess different functionalities due to their reactive layered structures, including impacting the thermal conductivity properties of the final clay composites $[15,16]$. When calcined, the innate structure of clays will be distorted [17], but the general layered structure is retained. This renders them susceptible to undergoing similar reactions as clays, but to varying effects. The effect of polymer addition on the thermal conductivity of calcined clay will thus be explored here.

Two common polymers in construction were selected for this purpose. They were polystyrene (PS) and polyethylene glycol (PEG), both possessing low thermal conductivities. They differed in that PS was a hydrophobic polymer, whereas PEG was a hydrophilic polymer (Figure 1). Their effects on the thermal property of calcined clays and the mechanism driving the results through understanding of polymer-watercalcined clay interaction will be discussed.

\section{Materials and Methods}

2.1. Clay and Polymer Materials. Two calcined clays obtained from Saint-Gobain Weber, Norway, were employed as per supplied. The calcined clays were rich in kaolinite (CK) and smectite (CS). CK was prepared by calcining at $800^{\circ} \mathrm{C}$ and it contained $47 \%$ kaolinite, $34 \% \mathrm{~K}$-feldspar, $17 \%$ quartz, and a minor amount of illite. CS was calcined at $850^{\circ} \mathrm{C}$ and contained $>50 \%$ smectite, $\sim 25 \%$ calcite, $\sim 8 \%$ kaolinite, $4 \%$ quartz, $3 \%$ siderite, and $1 \%$ pyrite. Both calcined clays contained $\sim 50 \%$ non-clay based materials, which were classified as "contaminants" in this investigation. The reason for using clay samples with similar amounts of contaminants was to ensure a good quantitative comparison between the two clays. More information on their hydrating properties and mechanical strength behaviors can be found in [18]. Two pure bentonites, a sodium bentonite $(\mathrm{NB})$ and a potassium bentonite $(\mathrm{KB})$, were included as references. The purpose of including them was to define the upper $(\mathrm{NB})$ and lower $(\mathrm{KB})$ limits for the incorporation of polymers and water by the clays. Bentonite was an expanding clay. Therefore, both water and polymers can adsorb both onto the clay surfaces and intercalate between the layered structures, whereby up to four layers of water molecules can be incorporated into the NB interlayer spacing. However, due to incorporation of $\mathrm{K}$ ions between the interlayer spacings, adsorption of polymers and water molecules was restricted to only the outer surfaces of clays for $\mathrm{KB}$.

Reagent-grade styrene (99\%) and polyethylene glycol (PEG, $\mathrm{Mw} \approx 400$ ) from EMD Millipore Corporation and potassium persulfate (KPS, 99\%) from Sigma-Aldrich were used without further purification.

2.2. Formation of Polymers-Calcined Clays Complexes. All four clay samples were employed in one-pot synthesis with the polymers to form polymer (calcined)-clays complexes (PCCs). NB and KB were selected as the model systems.

2.2.1. PS-PCC Synthesis. $15.4 \mathrm{~g}$ of a $1.3 \mathrm{wt}$.\% KPS solution was added to $178 \mathrm{~g}$ of a $10 \mathrm{wt} . \%$ styrene solution under stirring conditions. The temperature was increased and maintained at $70.0 \pm 0.1^{\circ} \mathrm{C}$ for $3 \mathrm{~h} .22 \mathrm{~g}$ of $9 \mathrm{wt} . \%$ (calcined) clay suspension was added to the reaction pot at $1 \mathrm{~h}$ and $2 \mathrm{~h}$ after temperature stabilized. The reaction products are denoted as PS1h and PS2h, respectively.

2.2.2. PEG-PCC Synthesis. A saturated solution of PEG was prepared with $60 \mathrm{~g}$ of $\mathrm{PEG}$ at $25^{\circ} \mathrm{C}$ to limit the amount of water for intercalating into the clay layers, while maximizing polymer dispersion. $24 \mathrm{~g}$ of (calcined) clay was then added and stirred for $1 \mathrm{~h}$.

For preparation of water saturated samples, the (calcined) clay samples were submerged in water under stirring condition for 15 minutes before drying.

All PCC samples were cooled to room temperature and centrifuged at $8000 \mathrm{rpm}$ for $10 \mathrm{~min}$. The final residue was dried in the oven at $80^{\circ} \mathrm{C}$ for 3 days except for NB samples. There, drying was prolonged to 2 weeks due to the high retention of water. The seemingly low temperature of drying at $80^{\circ} \mathrm{C}$ in contrast to at $>100^{\circ} \mathrm{C}$ was to ensure the stability of the polymer-clay complex. Prolonging drying up to 2 weeks was employed to compensate for the speed of drying.

2.3. Characterization of PCCs. Two characterization methods were employed here: hot disk thermal analysis for measurement of thermal conductivity and Fourier Transform Infrared (FTIR) spectroscopy for determining the chemical environment of bonds, particularly pertaining to $\mathrm{O}-\mathrm{H}$ functional groups within the samples.

Thermal conductivity was measured using a Hotdisk Thermal Constants Analyzer TPS 2500S. The Hotdisk has an accuracy better than 5\%. A transient plane source technique was applied $[19,20]$ and approximately $10 \mathrm{~g}$ of the samples was measured with a Kapton sensor radius $=3.189 \mathrm{~mm}(\# 5465)$. The plastic-film-covered sensor was sandwiched in between two powder samples and acted as a heat source in addition to 


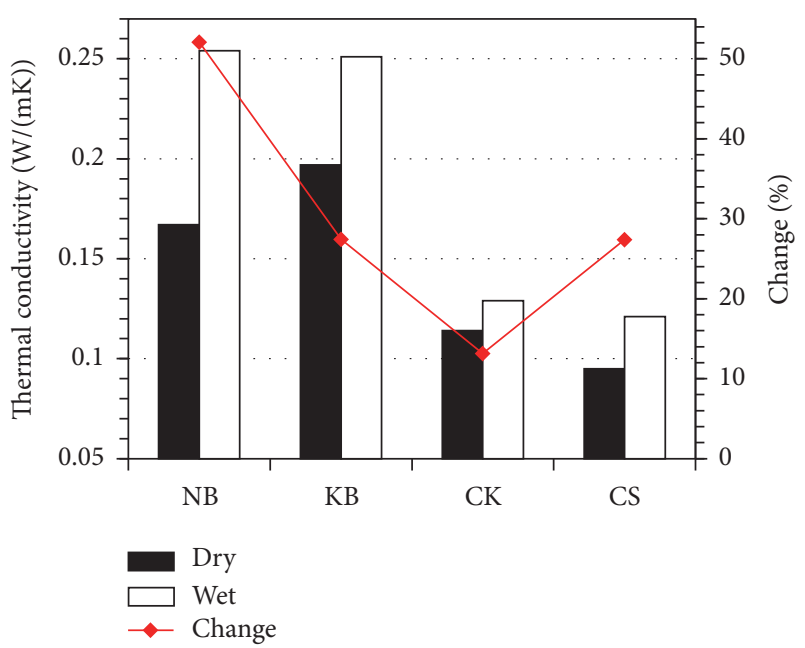

FIgURE 2: Thermal conductivity of dry and wetted (calcined) clay samples, with percentage difference of thermal conductivity values in dry and wetted samples represented as line figure (red).

registering the temperature increase in the samples. The temperature increase over time was recorded and used to calculate the thermal conductivity of the AIM samples. The heating power and heating time were varied independently to obtain the most appropriate testing conditions for each sample. The parameters were as follows: heating power was $0.1-0.7 \mathrm{~W}$, heating duration was $120-240 \mathrm{~s}$, and the final reported data were given as the arithmetic mean of 3 to 5 individual results. All error bars were calculated as $2 \mathrm{x}$ standard deviation of the mean values (i.e., $95.45 \%$ confidence interval).

FTIR measurements were conducted with a Thermo Nicolet 8700 FTIR spectrometer possessing a Smart Orbit accessory, which is a horizontal attenuated total reflectance (ATR) accessory (single reflection) with a diamond crystal, in an atmosphere with minimalized $\mathrm{CO}_{2}$ and $\mathrm{H}_{2} \mathrm{O}$ content through purging by a Parker Balston 74-5041 FTIR Purge Gas Generator. Approximately 2-4g of the samples was employed for each measurement. Each FTIR spectrum was performed with 32 scans at a resolution of $4 \mathrm{~cm}^{-1}$ and at least three repetitions of the same sample were recorded. The FTIR spectra given in this work were not ATR corrected, neither with respect to penetration depths nor with respect to absorbance band shifts. The measurement range was between $400 \mathrm{~cm}^{-1}$ and $4000 \mathrm{~cm}^{-1}$.

\section{Results and Discussion}

3.1. Effect of Water Addition on Thermal Conductivity of Clays without Polymers. Water is the main medium in a concrete mix and it greatly influences the thermal conductivity of materials. Therefore, the effect of water on the thermal conductivity values of clays, both calcined and pure bentonites, was first determined (Figure 2). The thermal conductivities of the dry clay samples were as follows: $\mathrm{KB}$ with $0.197 \mathrm{~W} /(\mathrm{mK})$, $\mathrm{NB}$ with $0.167 \mathrm{~W} /(\mathrm{mK}), \mathrm{CK}$ with $0.114 \mathrm{~W} /(\mathrm{mK})$, and CS with $0.095 \mathrm{~W} /(\mathrm{mK})$. The first observation was that calcined clay samples displayed much lower thermal conductivities than the model clay samples. This could arise from the amorphosity of the calcined clay. Upon calcination, the interlayer spaces of the clays were distorted [17]. This distortion may restrict the path of heat transfer through the formation of thermally insulating pockets or nanospaces. Another potential explanation for the lower thermal conductivity of calcined clays as compared to model clay samples may be due to the presence of other minerals as contaminants in the calcined clay samples. This indicated that the thermal conductivity of the calcined clay samples may differ when different raw materials with different amounts of contaminants are present. The composite matrix may affect heat transfer within the sample.

Upon wetting and subsequent drying to remove free water, the thermal conductivity of all clay samples was remeasured. Relative to the dry samples, the thermal conductivity values of all clay samples were higher after wetting. This could be attributed to the ability of the clay to stabilize and physically bind thermally conducting water onto the surfaces of and in between the layered structures of the clay particles. The order of increment in thermal conductivities was as follows: $\mathrm{CK}<\mathrm{CS} \approx \mathrm{KB}<\mathrm{NB}$, starting from an increment of $13 \%$ to $27 \%$ and finally $52 \%$. The order of variation in thermal conductivity may be explained by the inherent properties of the different clay samples. Both NB and $\mathrm{KB}$ were smectite based clays; that is, they possessed expanding interlayer spacing, which could adsorb up to four layers of water molecules [21] through swelling mechanism. As a result, high uptake of water onto the clay surfaces and in the interlayer spacing was expected, resulting in a significant change in thermal conductivity for the NB sample. The presence of $\mathrm{K}^{+}$in $\mathrm{KB}$ samples on the other hand restricted intercalation of water molecules into the layered surfaces. This can be attributed to the ideal geometric fitting of $\mathrm{K}^{+}$to the surface oxygen hexagons of the main layer, which reduced the hydration energy and entropically stabilized the interlayer spacing. In this way, water molecules mainly anchored onto the external surfaces of clay layers in the KB sample, resulting in overall lower uptake of water than NB.

CS consisted mainly of calcined smectite, an expanding clay, and thus it possessed similar properties to NB. As a result, the water retention capacity of CS can be correlated to the amount of smectite clay present in the CS sample. The variation in sorbed water as reflected by the change in thermal conductivity value was slightly lower than $50 \%$ of that of the NB sample, even when CS contained more than $50 \%$ smectite. This signified that while water sorption behavior by CS was similar to NB, a slight decrease in water uptake by the clay components after calcination may have occurred, assuming that contaminants in CS played an insignificant role in retaining water after drying. The difference in water affinity between calcined smectite in CS and normal smectite may have resulted from the alteration of the $\mathrm{Al}$ layers (conversion of 6-coordinated Al to 4-coordinated), partial oxidation of Fe, and reduction in Al-O-Si/Al-O-Al bonds [19]. This increased the amorphosity of the calcined smectite in CS through disruption of the layered structure of clays and hindered the sorption of water. 


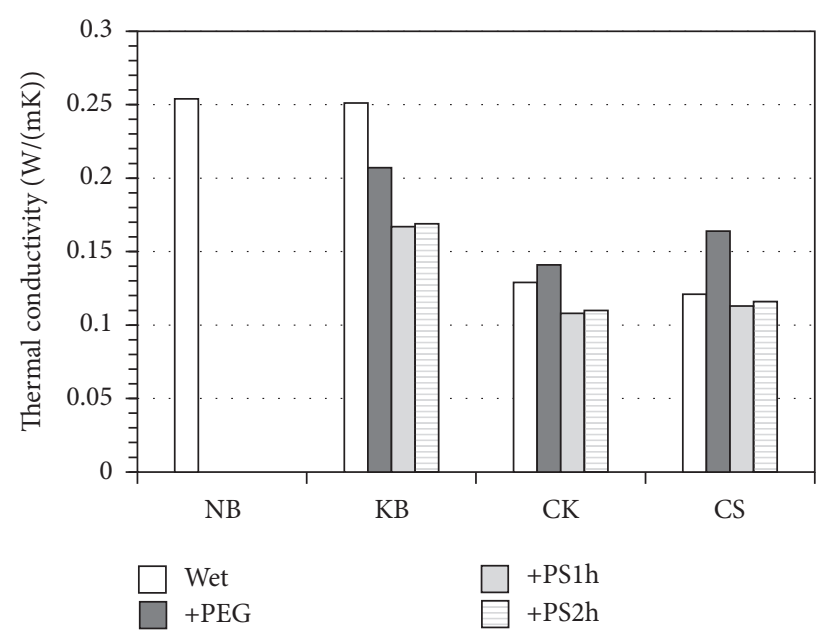

FIGURE 3: Thermal conductivity of clay samples, with and without polymers.

In the case of $\mathrm{CK}$, the dominating clay present was kaolin, a nonexpanding 1:1 layered structure. Due to its nonexpanding nature, the sorption of water by kaolin was limited to surface sorption, similar to $\mathrm{KB}$. The overall water affinity of $\mathrm{CK}$, which can be defined as proportional to the kaolin content $(\sim 50 \%)$, was thus significantly lower than the other clay samples, rendering a much lower change in thermal conductivity.

\subsection{Effect of PS and PEG on the Thermal Conductivity of Clays.} In applications, cement samples are always in a hydrated state. Therefore, the wetted clay samples were taken as the reference points. Upon addition of polymers, no thermal conductivity values with NB-polymer samples were measureable as these samples remained wet even after 2 weeks of drying. XRD analysis of the samples however displayed expanded clay interlayer spacing, attributing the wetness of polymers-NB to the strong stabilization of the polymers with attached water within the interlayer spaces. The strong retention of water molecules in the system rendered them unsuitable for reducing thermal conductivities of construction elements.

When $\mathrm{KB}$ was employed, the thermal conductivity of polymer-KB was lower than that of the wetted $\mathrm{KB}$ sample (Figure 3). The lower thermal conductivity values indicated displacement of sorbed water molecules by both the hydrophobic and the hydrophilic polymers from the clay surfaces. The change in thermal conductivity values after incorporating polymers was $-33 \%$ and $-17 \%$ for PS and PEG, respectively, indicating that water displacement may be higher when hydrophobic PS was employed as compared to PEG. This could potentially be explained by the water-seeking nature of polyethylene glycol. The $\mathrm{OH}$ functional groups in PEG seek and retain water, resulting in overall retention of water by the PEG polymer anchored on the mineral. On the other hand, due to the hydrophobicity of PS, association with water was minimum, and thus little water was trapped within the polymer-clay complex. Another observation was the effect of synthesis duration for KB-polystyrene. Insignificant

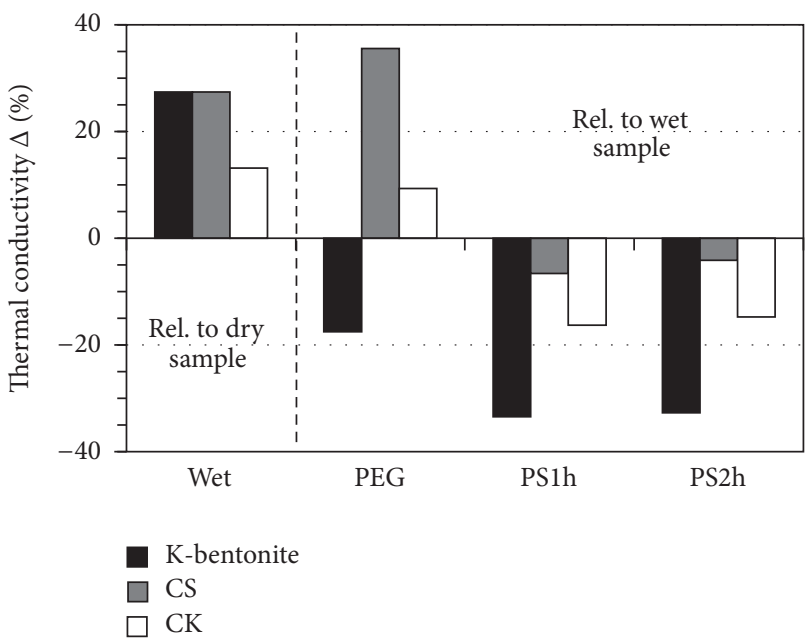

Figure 4: Percentage change in thermal conductivity of clays containing polymers relative to the wetted samples.

differences in the thermal conductivity values could be observed for the two synthesis methods for KB-polystyrene, indicating that synthesis of this PS-KB is a robust pathway.

When the polymers PEG and PS were added to the two calcined clays, a slightly different trend was observed. Similar to KB samples, the addition of hydrophobic PS to the calcined clay samples lowered the thermal conductivity values of the samples. However, the impact of PS addition on thermal conductivity values of calcined clay (7\% and $16 \%$ decrease) was less pronounced than that of bentonite (33\%, Figure 4), possibly related to dilution effect resulting from the presence of contaminants in the sample. On the other hand, in the presence of PEG, thermal conductivity values of PEG-CS and PEG-CK were 35\% and 9\% higher than the wetted calcined clay samples, contrary to observation of KB sample.

To understand the thermal conductivity values of PCC samples, clarification on the effect of polymer/water/calcined clay interactions was sought by performing FTIR analysis on the dried polymer-calcined clay samples. Figure 5 presents the chemical environment of the $\mathrm{O}-\mathrm{H}$ bonds present in the polymer-calcined clay (PCC) samples. When PS was added, no stretching or vibrating bends for $\mathrm{O}-\mathrm{H}$ bond can be observed in the FTIR spectra, regardless of clay types. This indicated that bound water was absent from these samples, whereby hydrophobic PS demotes the sorption of water by calcined clay, resulting in little influence of bound water on the thermal conductivity values of the PCC samples.

On the other hand, $\mathrm{O}-\mathrm{H}$ stretching and bending bonds were observed when PEG was present. Asymmetric O-H stretching bond shifted upfield from $3319 \mathrm{~cm}^{-1}$ of pure water to $3394 \mathrm{~cm}^{-1}$. Symmetric O-H stretching shifted downfield from $3246 \mathrm{~cm}^{-1}$ to $\sim 2900 \mathrm{~cm}^{-1}$. The presence of two $\mathrm{O}-\mathrm{H}_{\text {sym }}$ stretching bonds in the PEG-calcined clay sample indicated that the $\mathrm{O}-\mathrm{H}$ bonds were present in two environments, most likely arising from the inherent $\mathrm{O}-\mathrm{H}$ bond of the PEG and the $\mathrm{O}-\mathrm{H}$ bond from the attached water. Directly bound water molecules to the PS-PCC samples are unlikely as no $\mathrm{O}-\mathrm{H}$ bonds were detected in these samples. 


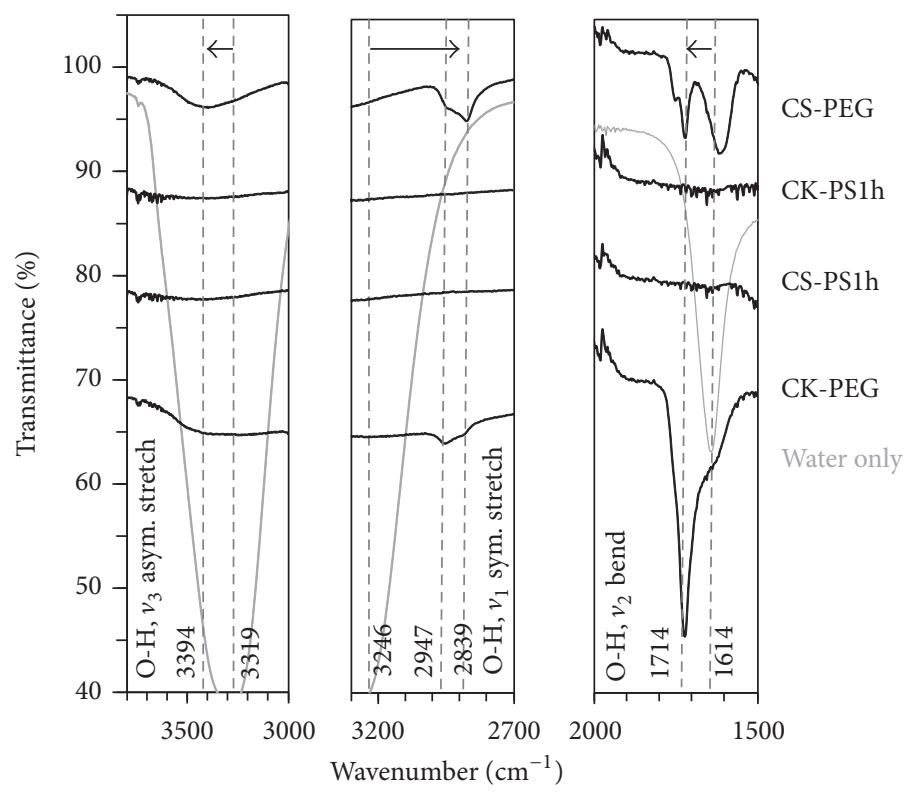

FIGURE 5: FTIR spectra of CS-PEG, CS-PS1, CK-PEG, CK-PS1, and water. PS2h spectrum was identical to PS1h spectrum. Stretching $\left(\sim 3500-2900 \mathrm{~cm}^{-1}\right)$ and bending $\left(1700-1600 \mathrm{~cm}^{-1}\right)$ bands of O-H in water molecules affected by PEG-clay were observed.

A closer look at the group of peaks $\sim 1700 \mathrm{~cm}^{-1}$ indicated that $\mathrm{O}-\mathrm{H}$ bonds were present in multiple different environments. Particularly, it can be postulated that the water molecules had more degrees of freedom in $\mathrm{CK}$ than that in CS due to the strong intensity of $\mathrm{O}-\mathrm{H}$ bend in $\mathrm{CK}-\mathrm{PCC}$ samples at $1714 \mathrm{~cm}^{-1}$, which was absent in CS-PCC samples. This indicated that mode of PEG-water uptake by CS may be driven mainly by intercalation of hydrated $\mathrm{PEG}$, resulting in low intensity of $\mathrm{O}-\mathrm{H}$ bending due to the constraint in the interlayer spacing between clay layers for strong $\mathrm{O}-\mathrm{H}$ vibration. On the other hand, as hydrated PEG was mainly adsorbed onto the surface of $\mathrm{CK}$, the water molecules were more "free."

The results here thus demonstrate three points in relation to the thermal conductivity values of the samples: (1) the water affinity of the PEG sample whereby water molecules were trapped within the interpores of the calcined clays by association with the hydrophilic PEG increased the thermal conductivity of the PCC sample. (2) Hydrophobic PS, on the other hand, does not retain water, and thus addition of PS decreased the thermal conductivity of the calcined clay sample. (3) Due to the difference in clay structure between CK and CS, the modes of PEG and water uptake differ, which may result in higher water uptake and thus higher thermal conductivity values for CS due to greater stability in interaction between polymer and calcined clay.

\section{Conclusions}

Calcined clays had been previously shown to be an effective substituent binder for cement in lowering the thermal conductivity without compensating the mechanical performances. In this work, we investigated the possibility of further lowering the thermal conductivity of calcined clays through the incorporation of polymers. Calcined clays behaved similarly to clays in their interactions with polymers and water. However, the presence of contaminants played a role in impacting the overall thermal conductivity of the calcined clays. Due to the inherent nanostructure of clays and their water affinity tagged to the polymers, polyethylene glycols were found to be unsuitable for reducing thermal conductivity values of calcined clays, particularly for samples based on smectites. On the other hand, polystyrene can effectively decrease the thermal conductivity values of calcined clays, regardless of clay type, due to their low affinity for water. This study indicated that hydrophobic polymer was more suitable to improve the thermal conductivity values of calcined clays.

As an outlook, further understanding of the mechanistic correlation between porosity of these nanomaterials and the resulting thermal conductivities, particularly with relation to their mode of heat transfer (convection or conduction), of the samples can be probed. From a practical point of view, larger amounts of polystyrene-calcined clay samples need to be produced, especially at a balance with, for example, mechanical properties. The inherent characteristic of the raw clay must be taken into account, particularly the clay type and amount of contaminant. The results obtained can be further extended to large-scale testing on concrete construction containing reinforcement bars for determination of actual application effectivity. Nevertheless, this current investigation demonstrated the feasibility of generating more thermally insulating materials at a low cost.

\section{Conflicts of Interest}

The authors declare that there are no conflicts of interest regarding the publication of this paper. 


\section{Acknowledgments}

This work has been funded by the Concrete Innovation Centre (COIN) and the Research Centre on Zero Emission Buildings (ZEB). Linn Ingunn Christie Sandberg is acknowledged for her help with laboratory tasks.

\section{References}

[1] B. P. Jelle, A. Gustavsen, and R. Baetens, "The path to the high performance thermal building insulation materials and solutions of tomorrow," Journal of Building Physics, vol. 34, no. 2, pp. 99-123, 2010.

[2] R. Sinha, M. Lennartsson, and B. Frostell, "Environmental footprint assessment of building structures: a comparative study," Building and Environment, vol. 104, pp. 162-171, 2016.

[3] R. Baetens, B. P. Jelle, J. V. Thue et al., "Vacuum insulation panels for building applications: a review and beyond," Energy and Buildings, vol. 42, no. 2, pp. 147-172, 2010.

[4] S. E. Kalnæs and B. P. Jelle, "Vacuum insulation panel products: a state-of-the-art review and future research pathways," Applied Energy, vol. 116, pp. 355-375, 2014.

[5] R. Baetens, B. P. Jelle, A. Gustavsen, and S. Grynning, "Gasfilled panels for building applications: a state-of-the-art review," Energy and Buildings, vol. 42, no. 11, pp. 1969-1975, 2010.

[6] R. Baetens, B. P. Jelle, and A. Gustavsen, "Aerogel insulation for building applications: a state-of-the-art review," Energy and Buildings, vol. 43, no. 4, pp. 761-769, 2011.

[7] R. Baetens, B. P. Jelle, and A. Gustavsen, "Phase change materials for building applications: a state-of-the-art review," Energy and Buildings, vol. 42, no. 9, pp. 1361-1368, 2010.

[8] L. Ratke, "Fabrication and properties of a new light weight concrete: aerogel concrete," Beton- und Stahlbetonbau, vol. 103, no. 4, pp. 236-243, 2008.

[9] T. Gao, B. P. Jelle, A. Gustavsen, and S. Jacobsen, "Aerogelincorporated concrete: an experimental study," Construction and Building Materials, vol. 52, pp. 130-136, 2014.

[10] S. Ng, B. P. Jelle, and T. Stæhli, "Calcined clays as binder for thermal insulating and structural aerogel incorporated mortar," Cement and Concrete Composites, vol. 72, pp. 213-221, 2016.

[11] J. A. Forrester, "Burnt clay pozzolans," in Proceedings of the Meeting on Small-Scale Manufacture of Cement Materials, pp. 53-59, Inter-Med Technologies, London, UK, 1974.

[12] H. Justnes, T. Østnor, and S. Ng, "Applicability of Nordic clays as SCM," in Proceedings of the International RILEM Conference on Materials, Systems and Structures in Civil Engineering Conference Segment on Concrete with Supplementary Cementitious materials, Technical University of Denmark, Lyngby, Denmark, August 2016.

[13] A. R. Bahramian, L. S. Ahmadi, and M. Kokabi, "Performance evaluation of polymer/clay nanocomposite thermal protection systems based on polyethylene glycol phase change material," Iranian Polymer Journal, vol. 23, no. 3, pp. 163-169, 2014.

[14] T. A. Østnor, H. Justnes, and S. R. Rudolfsen, "Strength development and durability of mortars with calcined marl as supplementary cementing material," in Proceedings of the 1st International Conference on Concrete Sustainability, pp. 567572, Tokyo, Japan, May 2013.

[15] H. Zhou, S. Zhang, and M. Yang, "Modeling the thermal conductivity of exfoliated polymer/clay nanocomposites," Journal of Applied Physics, vol. 102, no. 8, Article ID 084312, 2007.
[16] P. Meneghetti and S. Qutubuddin, "Synthesis, thermal properties and applications of polymer-clay nanocomposites," Thermochimica Acta, vol. 442, no. 1-2, pp. 74-77, 2006.

[17] T. Danner, H. Justnes, G. Norden, and T. Østnor, "Feasibility of calcined marl as an alternative pozzolanic," in Proceedings of the 1st International Conference of Calcined Clays for Sustainable Concrete, K. Scrivener and A. Favier, Eds., vol. 10 of RILEM Book, pp. 67-73, Springer, Lausanne, Switzerland, 2015.

[18] T. A. Østnor and H. Justnes, "Durability of mortar with calcined marl as supplementary cementing material," Advances in Cement Research, vol. 26, no. 6, pp. 344-352, 2014.

[19] D. P. Bentz, "Transient plane source measurements of the thermal properties of hydrating cement pastes," Materials and Structures, vol. 40, no. 10, pp. 1073-1080, 2007.

[20] S. E. Gustafsson, "Transient plane source techniques for thermal conductivity and thermal diffusivity measurements of solid materials," Review of Scientific Instruments, vol. 62, no. 3, pp. 797-804, 1991.

[21] F. Bergaya and G. Lagaly, "General introduction: clays, clay minerals, and clay science," in Handbook of Clay Science, F. Bergaya, B. K. G. Theng, and G. Lagaly, Eds., vol. 1 of Developments in Clay Science, Elsevier, Amsterdam, the Netherlands, 2013. 

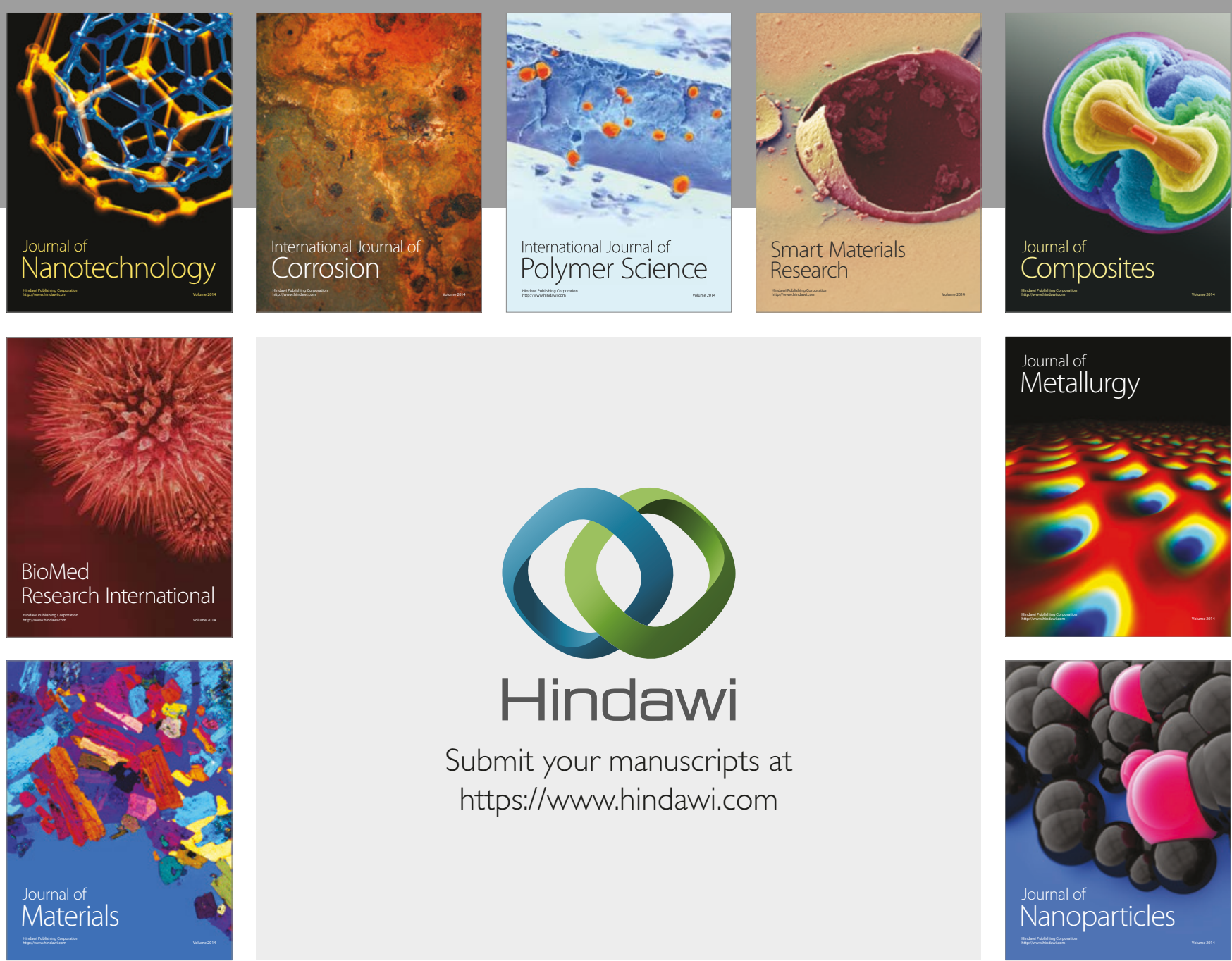

\section{Hindawi}

Submit your manuscripts at

https://www.hindawi.com
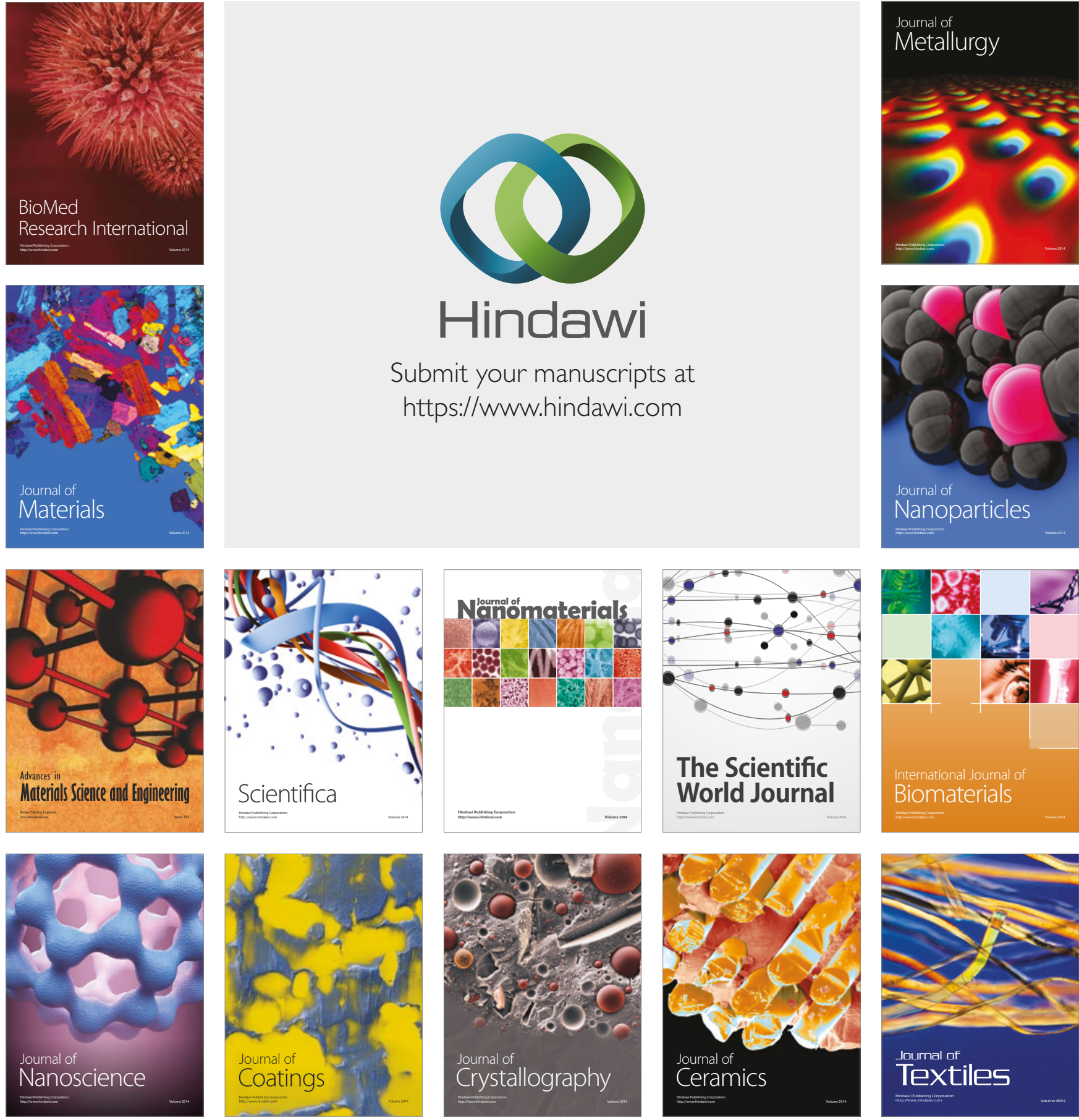

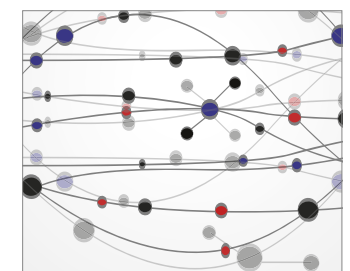

The Scientific World Journal
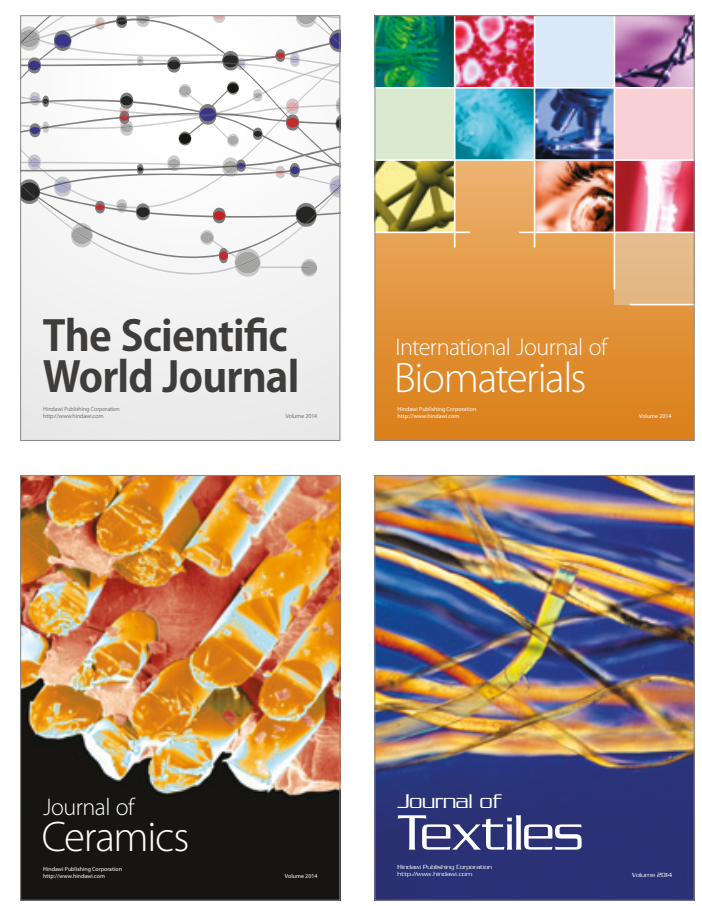